\title{
Development of an empirical interatomic potential for the Ag-Ti system
}

\author{
Ying Zhou ${ }^{\mathrm{a}, *}$, Roger Smith ${ }^{\mathrm{a}}$, Steven D. Kenny ${ }^{\mathrm{b}}$, Adam L. Lloyd ${ }^{\mathrm{a}}$ \\ ${ }^{a}$ Department of Mathematical Sciences, Loughborough University, Leicestershire, LE11 3TU, United Kingdom \\ ${ }^{b}$ Department of Materials, Loughborough University, Leicestershire, LE11 3TU, United Kingdom
}

\begin{abstract}
Two interatomic potential mixing rules for the Ti-Ag system were investigated based on the embedded-atom method (EAM) elemental potentials. First principles calculations were performed using SIESTA for various configurations of the $\mathrm{Ti}-\mathrm{Ag}$ system to see which model best fitted the $a b$ initio results. The results showed that the surface energies, especially that of Ti, were not well fitted by either model and the surface binding energies differed from the $a b$ initio calculations. As a result, the modified embedded-atom method (MEAM) was investigated. In contrast to the other models, surface energies for pure Ti calculated by MEAM were in good agreement with the experimental data and the $a b$ initio results. The MEAM mixing rule was used to investigate Ag ad-atoms on Ti and Ti ad-atoms on Ag. The results showed good agreement with SIESTA after parameter optimisation.
\end{abstract}

Keywords: titanium, silver, semi-empirical potential, density-functional calculations

\section{Introduction}

Multilayer thin film stacks are used in the glass industry where the different layers are usually produced using magnetron sputtering. In this process low energy particles impact on a substrate to form the thin film. Silver is used as an infra red blocker in the multilayer structure and one interface of interest is that between layers of silver and titanium. To investigate the deposition process, an accurate atomistic model of the $\mathrm{Ti}-\mathrm{Ag}$ system, which reproduces surface properties well, is required. To date many models of binary metal systems have been concerned with bulk alloy properties.

There are a few existing semi-empirical elemental potentials for $\mathrm{Ti}$ and $\mathrm{Ag}$, including the embedded-atom method (EAM) or the modified embedded-atom method (MEAM). To model binary systems, various mixing rules have been proposed. Using the EAM approach, we examined two of these rules due to Johnson [1] and Ward [2] using the elemental potentials from Wadley 3]. We also examined EAM potentials by Ackland [4, 5] and Mishin [6, 7] for pure Ag and Ti for the surface properties, but did not apply the mixing rules on these. In the case of MEAM [8], the elemental potentials for $\mathrm{Ti}$ and $\mathrm{Ag}$ are $2 \mathrm{NN}$. MEAM has its own in built mixing rules but has various free parameters which can be optimised. We developed a new MEAM potential for $\mathrm{Ti}-\mathrm{Ag}$, where these parameters were optimised especially for the surface structures.

\footnotetext{
* Corresponding author

Email address: y.zhou3@lboro.ac.uk (Ying Zhou)
}

\section{Methodology}

\subsection{Embedded-atom method}

The embedded-atom method for pure metals was first introduced by Daw and Baskes in 1984 [9, 10], and also in that year, Finnis and Sinclair 11] proposed a similar description. Both methods consist of two parts: a many body term and a conventional pair-potential term. The generic form of the potential can be expressed as:

$$
E_{t o l}=\frac{1}{2} \sum_{i \neq j} \phi\left(r_{i j}\right)+\sum_{i} F_{i}\left(\rho_{i}\right),
$$

where $\phi_{i j}$ is the pairwise interaction between atoms $i$ and $j$ with the separation of $r_{i j}$, and $F_{i}$ is the embedding energy of atom $i . \quad \rho_{i}$ is the electron density of atom $i$ at the site. Johnson developed a generic mixing rule which uses a modified pair potential and electron density terms from the elemental values [1].

For the Ward mixing rule a new pair potential for atoms of different species was also constructed but the electron density term and embedding function were scaled versions of the elemental functions [2].

\subsection{Modified embedded-atom method}

First proposed by Baskes [8] as a modification to the EAM, the MEAM has been applied to many pure materials and parameters for some binary and ternary systems have also been developed. The total energy of a system is expressed as :

$$
E_{t o l}=\sum_{i}\left[F_{i}\left(\bar{\rho}_{i}\right)+\frac{1}{2} \sum_{j(\neq i)} S_{i j} \phi\left(R_{i j}\right)\right],
$$


where $S_{i j}$ is the many body screening function between $i$ and $j$ and is calculated as the product of the screening factors $S_{i k j}$. The MEAM is angular-dependent, which means that the computation of background electron density considers the direction of the bonding. Screening parameters in the pair interaction and background electron density are used. Ellipses are constructed of the form

$$
x^{2}+\frac{1}{C} y^{2}=\left(\frac{1}{2} R_{i j}\right)^{2} .
$$

If in any $(i-k-j)$ interaction, an atom $k$ lies outside of the ellipse defined by $C_{\max }$ then atom $k$ is assumed to be screened and pair $i-k$ interaction is ignored. If atom $k$ lies within the ellipse defined by $C_{\text {min }}$ then the i-j interaction is screened. The original MEAM [8, only considered the first-nearest neighbours in the embedding function so that the screening effect had to be strong to compensate for the energy. However, some stability problems occurred for the cubic and hexagonal closed pack (hcp) structures. Later the second even the third nearest neighbours are partially considered in order to overcome these shortcomings [12] . Therefore the screening effects are reduced and less severe.

\subsection{Density functional theory}

First principles calculations were performed using the density functional theory (DFT) with the SIESTA code [13. We use the generalized gradient approximation (GGA) with the Perdew, Burke and Ernzerhof (PBE) [14] for the exchange and correlation functional. The bases and pseudopotential for Ti are from 15. The DFT results for Ag were explored by Lloyd [16].

A 500 Ry grid cutoff is employed for the pure titanium structures (bcc, fcc and hcp) and the Monkhorst-Pack kpoint meshes are $16 \times 16 \times 16$ and $12 \times 12 \times 12$ for bcc and fcc structures respectively. For the Ag and Ti slabs, a $3 \times 3 \times 1 \mathrm{k}$-point sampling is used and the mesh cutoff is $250 \mathrm{Ry}$ in order to save computation time. The Ag (100) surface consists of 18 atoms and the Ag (111) surface has 20 atoms for each layer. The Ti basal plane has 20 atoms per layer. All slabs consist 6 layers.

\section{Optimisation of potential parameters}

A 2NN MEAM potential for both Ti [17] and Ag [12] is chosen for the elemental potentials. For our Ag-Ti binary system, the ordered bcc AgTi structure is first chosen as a reference. The cohesive energy $E_{c}$, nearest-neighbour distance $r_{e}$ and $\alpha$ (a parameter in the MEAM method, determined by the cohesive energy, bulk modulus and atomic volume) are calculated from SIESTA. In the original binary MEAM, $\alpha$ is taken as an average of the elemental $\alpha$ values but here we fit it from the SIESTA calculations. For the combined system the screening parameters, $C_{m i n}$, $C_{\max }$ can be used to optimise the potential properties along with electron density ratio between individual elements $\rho_{0}$. In previous fittings for the binary metallic system, the screening parameters are determined based on the values of pure materials without taking the surface structures into account. We fitted these nine parameters to the surface binding energies of 21 different configurations with one ad-atom and the distorted lattices (b11 AgTi and fcc $\left.\mathrm{AgTi}_{3}\right)$. The surface binding energy is calculated as:

$$
E_{\text {binding }}=E_{\text {total }}-E_{\text {slab }}
$$

To optimise the parameters an objective function was defined as the sum of the weighted squared deviation from the desired targets. Different weighting coefficients were assigned to give more prominence to the surface structures. When developing the MEAM for the Ti-O system, Joost [18] suggested that the downhill simplex method and the Powell method always trap the objective function in local minima. Therefore in our study the simulated annealing method was used first with multiple different initial guesses and temperature. After some sets of parameters were accepted, we used both the downhill simplex method and the Powell method to find the local minima of these sets of values. The optimised parameters (shown in Table 1) gives the least weighted error.

Table 1: MEAM parameters for the Ag-Ti system

\begin{tabular}{ll}
\hline Property & Value \\
\hline$E_{c}(e V /$ atom $)$ & 3.80 \\
$r_{e}(\AA)$ & 2.875 \\
$\alpha$ & 5.95 \\
$\rho_{0}^{A g}: \rho_{0}^{T i}$ & $1: 1$ \\
$C_{\min }(A g-A g-T i)$ & 0.67 \\
$C_{\max }(A g-A g-T i)$ & 2.39 \\
$C_{\min }(A g-T i-A g)$ & 0.3 \\
$C_{\max }(A g-T i-A g)$ & 2.15 \\
$C_{\min }(T i-A g-T i)$ & 1.44 \\
$C_{\max }(T i-A g-T i)$ & 2.80 \\
$C_{\min }(A g-T i-T i)$ & 0.37 \\
$C_{\max }(A g-T i-T i)$ & 0.54 \\
\hline
\end{tabular}

\section{Results}

The lattice constants for bcc, fcc and hcp Ti were computed first. The lattice constants for b11 AgTi and $\mathrm{c} 11_{b}$ $\mathrm{AgTi}_{2}$ are shown in Table 3.

\subsection{Surface energies}

Surface energies for both $\mathrm{Ti}$ and Ag were calculated and compared to the experimental results and $a b$ initio data. These energies are shown in Table 4. For Ti, the EAM calculations underestimate the surface energy but MEAM gives better agreement. The underestimation of surface energy may lead to the wrong prediction of the positions of the ad-atoms in the substrate. 
Table 2: Lattice constants for Ti predicted by SIESTA, previous DFT calculations, MEAM and EAM potentials.

\begin{tabular}{lcccc}
\hline Structure & bcc & fcc & \multicolumn{2}{c}{ hcp } \\
& $\mathrm{a}(\AA)$ & $\mathrm{a}(\AA)$ & $\mathrm{a}(\AA)$ & $\mathrm{c} / \mathrm{a}$ \\
\hline Expt.[19] & 3.26 & 4.10 & 2.95 & 1.588 \\
SIESTA & 3.31 & 4.19 & 2.98 & 1.599 \\
DFT[15] & - & - & 2.99 & 1.588 \\
MEAM & 3.27 & 4.13 & 2.945 & 1.592 \\
Mishin & 3.23 & 4.15 & 2.95 & 1.589 \\
Wadley & 3.29 & 4.16 & 2.94 & 1.622 \\
Ackland & 3.26 & 4.17 & 2.97 & 1.589 \\
\hline
\end{tabular}

Table 3: Lattice constants for b11 AgTi and $\mathrm{c} 11_{b} \mathrm{AgTi}_{2}$ predicted by SIESTA, EAM and MEAM.

\begin{tabular}{lcccc}
\hline & \multicolumn{2}{c}{$\mathrm{AgTi}$} & \multicolumn{2}{c}{$\mathrm{AgTi}_{2}$} \\
& $\mathrm{a}(\AA)$ & $\mathrm{c}(\AA)$ & $\mathrm{a}(\AA)$ & $\mathrm{c}(\AA)$ \\
\hline Expt.[20] & 4.10 & 4.08 & 4.19 & 11.85 \\
SIESTA & 4.16 & 4.20 & 3.33 & 10.43 \\
MEAM & 4.12 & 3.95 & 3.46 & 8.53 \\
Ward & 4.27 & 3.50 & 3.50 & 8.30 \\
Johnson & 4.21 & 4.25 & 3.63 & 8.65 \\
\hline
\end{tabular}

Table 4: Surface energies $\left(J / m^{2}\right)$ predicted by SIESTA, MEAM and EAM for $\mathrm{Ag}$ and Ti.

\begin{tabular}{llll}
\hline & $\mathrm{Ag}(100)$ & $\mathrm{Ag}(111)$ & $\mathrm{Ti}(0001)$ \\
\hline Expt.[21] & 1.246 & 1.250 & 2.100 \\
SIESTA & 0.972 & 0.982 & 2.381 \\
MEAM & $0.966\left(0.983^{a}\right)$ & $0.832\left(0.842^{a}\right)$ & $2.153\left(2.156^{b}\right)$ \\
Wadley & 0.979 & 0.907 & 1.285 \\
Ackland & $0.759\left(0.759^{c}\right)$ & $0.621\left(0.620^{c}\right)$ & $0.987\left(0.993^{d}\right)$ \\
Mishin & $0.940\left(0.940^{e}\right)$ & $0.862\left(0.862^{e}\right)$ & $1.265\left(1.725^{f}\right)$ \\
\hline
\end{tabular}

${ }^{a} \operatorname{Ref[12},{ }^{b} \operatorname{Ref}[17],{ }^{c} \operatorname{Ref}[4],{ }^{d} \operatorname{Ref[}[5],{ }^{e} \operatorname{Ref}\left[\underline{6},{ }^{f} \operatorname{Ref}[7]\right.$

\subsection{Surface binding energy}

The calculated surface binding energies are shown in Figure 1. From SIESTA we found that a Ti atom in the surface layer of both $\mathrm{Ag}$ (100) and $\mathrm{Ag}$ (111), with an displaced $\mathrm{Ag}$ ad-atom, is energetically preferable to being a $\mathrm{Ti}$ ad-atom on the surface. However, on the Ti (0001) plane, the configuration with the $\mathrm{Ag}$ ad-atom is more stable than the other case.

The binding energies calculated by the MEAM are in much better agreement with SIESTA than those from Johnson and Ward especially on the Ti surface. This is also true for $\mathrm{Ag}$ surfaces, when the Ti atom joins the surface layer.

\subsection{Single Point Deposition}

Since the motivation for the work is to use the potential for modelling thin film growth, Molecular Dynamics
(MD) simulations were carried out using in-house developed LBOMD package to model the impact of single atoms incident normally onto the different surfaces in the energy range $0.1-40 \mathrm{eV}$. The Ti substrate consisted of 10 layers with 100 atoms in each layer. The Ag (100) substrate consisted of 14 layers with 98 atoms in each layer. The $\mathrm{Ag}$ (111) substrate consisted of 12 layers with 88 atoms in each layer. In the simulation, 1000 uniformly generated grid points were used in each deposition area. The single atoms were deposited to the surfaces with kinetic energies at $0.1,1,10$ and $40 \mathrm{eV}$ from grid points $10 \AA$ above the substrate. The simulation was $10 \mathrm{ps}$ and timestep was $1 \mathrm{fs}$. The bottom layer of the lattice was fixed and the last but one layer kept heated to $300 \mathrm{~K}$ with a Berendsen thermostat. Four categories are summarised: "penetrate" means that the surface layer was penetrated by the impact atom and "surface layer" means the deposited atom became part of the surface layer. "adsorb" stands for the deposited atom being adsorbed on the first layer while "rebound" means that the atom is reflected.

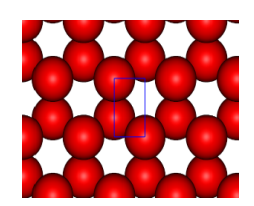

(a) $\mathrm{Ti}(0001)$

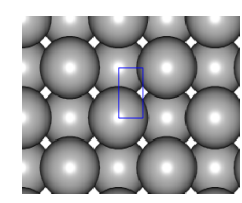

(b) $\mathrm{Ag}(100)$

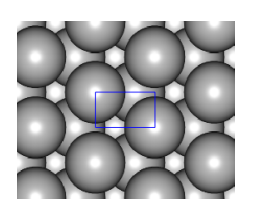

(c) $\mathrm{Ag}(111)$
Figure 2: Deposition areas chosen on Ti (0001) surface, Ag (100) and $\mathrm{Ag}$ (111) surfaces when impacting single atoms.

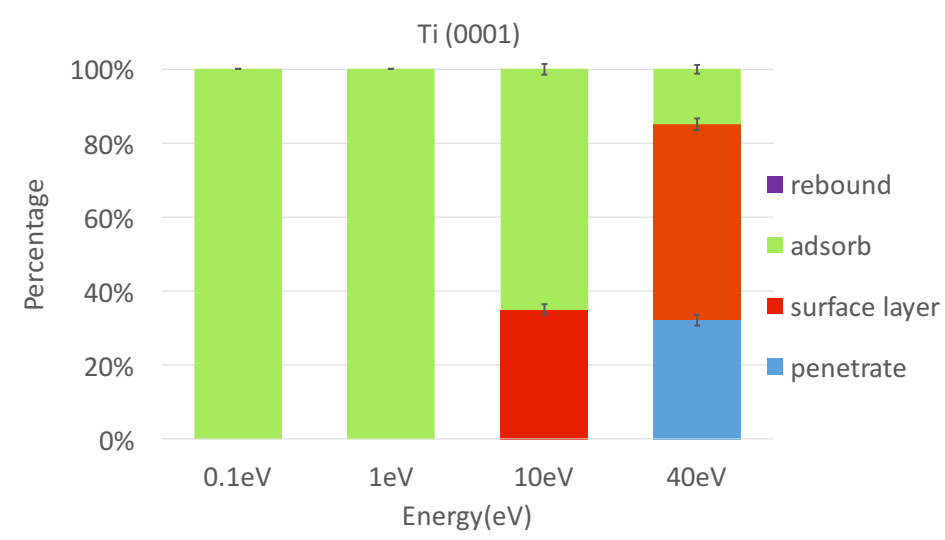

Figure 3: Results of depositing single $\mathrm{Ag}$ atoms onto perfect $\mathrm{Ti}$ (0001) surfaces. 


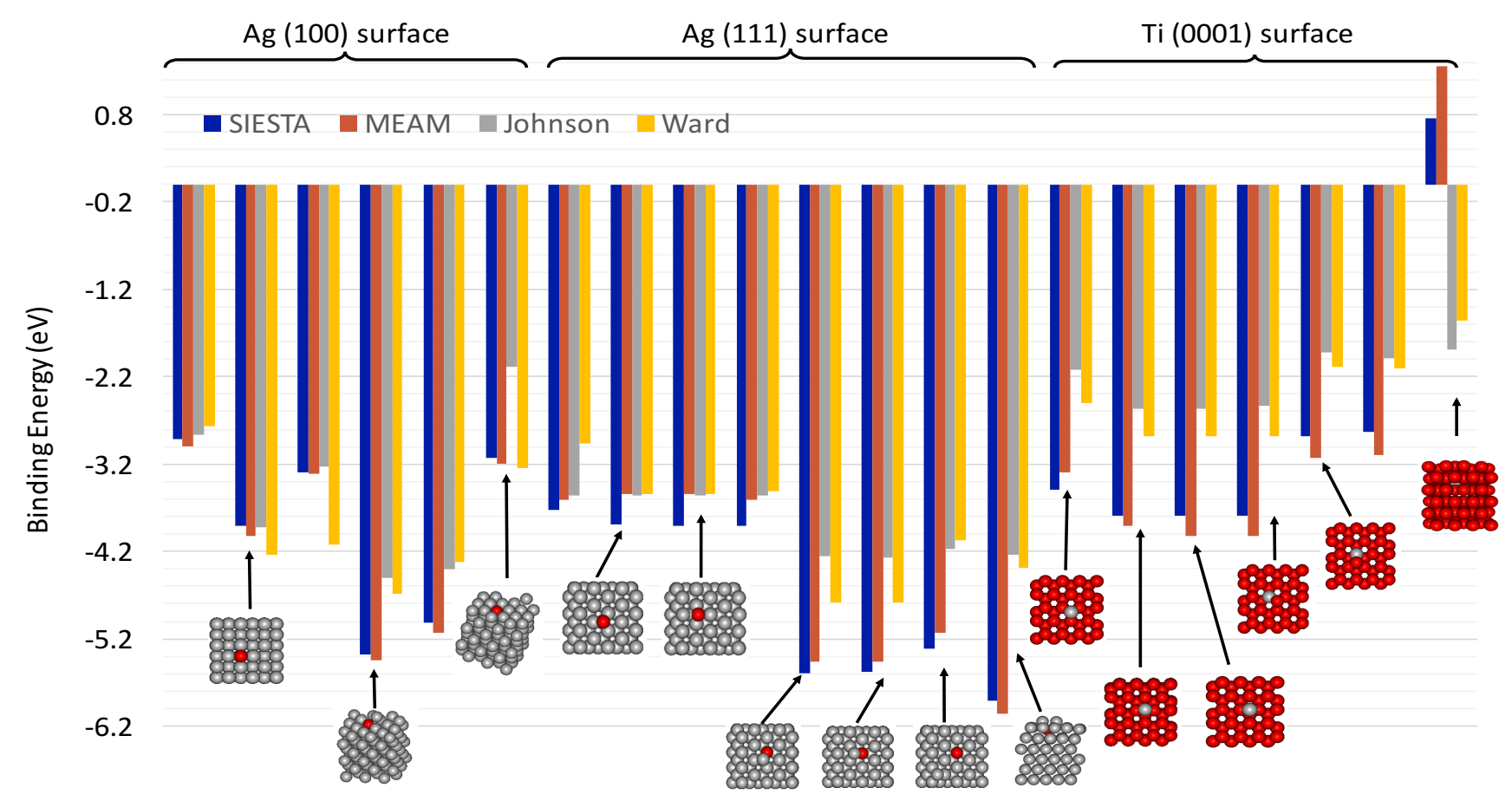

Figure 1: Surface binding energies for various structures of one ad-atom predicted by SIESTA, MEAM, Johnson and Ward. (For interpretation of the references to colour in this figure legend, the reader is referred to the web version of this article.)

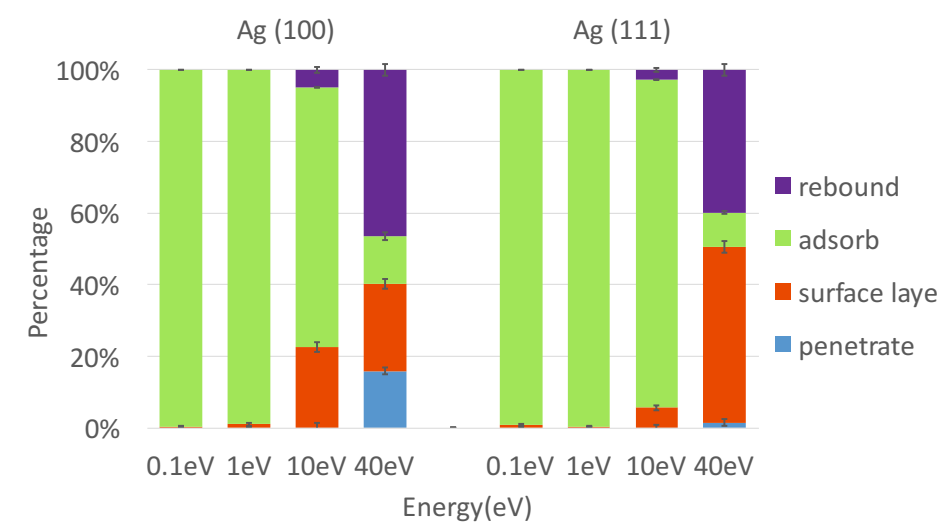

Figure 4: Results of depositing single Ti atoms onto perfect Ag (100) and Ag (111) surfaces.

The results shown in Figure 3 show that at low energies the $\mathrm{Ag}$ ad-atom resides on the surface while more atoms can penetrate the surface when the impact is above 10 $\mathrm{eV}$. Figure 4 shows that it is easier to penetrate the $\mathrm{Ag}$ (100) surface compared to Ag (111) and although it is energetically more favourable for a $\mathrm{Ti}$ atoms to join the Ag surface, only a few atoms join the surface layer at low energies, indicating that there is an energy barrier to be overcome. Even at $10 \mathrm{eV}$ most $\mathrm{Ti}$ atoms appear as adatoms on the surface and only when the energy is raised to $40 \mathrm{eV}$ do more Ti atoms join the surface.

\section{Summary}

The surface energy for Ti is underestimated by the EAM compared to experiment and ab initio calculations while the MEAM performs better. A new MEAM potential has been developed based on the existing MEAM elemental potentials for $\mathrm{Ag}$ and $\mathrm{Ti}$ which gives good agreement with the surface binding energies. We will use these parameters to simulate the surface growth for the Ag-Ti system. At normal incidence and impact energies less than $10 \mathrm{eV}$, typical for many magnetron sputter devices, the atoms prefer to deposit as ad-atoms on the surface.

\section{Acknowledgments}

This work has been supported by AGC Glass Europe and the Loughborough HPC unit. We would like to thank David Cornil and Jérôme Conil for helping us setup the SIESTA for the calculations of Ti. Our warm thanks go to Hugues Wiame and Benoit Lecomte for the helpful discussions. This work was funded by grant EP/K000055/1.

[1] R. Johnson, Physical Review B 39 (17) (1989) 12554.

[2] L. Ward, A. Agrawal, K. Flores, W. Windl, arXiv preprint arXiv:1209.0619.

[3] X. Zhou, R. Johnson, H. Wadley, Physical Review B 69 (14) (2004) 144113.

[4] G. Ackland, G. Tichy, V. Vitek, M. Finnis, Philosophical Magazine A 56 (6) (1987) 735-756.

[5] G. Ackland, Philosophical Magazine A 66 (6) (1992) 917-932.

[6] P. Williams, Y. Mishin, J. Hamilton, Modelling and Simulation in Materials Science and Engineering 14 (5) (2006) 817.

[7] R. Zope, Y. Mishin, Physical Review B 68 (2) (2003) 024102.

[8] M. Baskes, Physical Review B 46 (5) (1992) 2727. 
[9] M. Daw, M. Baskes, Physical Review B 29 (12) (1984) 6443.

[10] M. Daw, M. Baskes, Physical Review Letters 50 (17) (1983) 1285.

[11] M. Finnis, J. Sinclair, Philosophical Magazine A 50 (1) (1984) 45-55.

[12] B. Lee, J. Shim, M. Baskes, Physical Review B 68 (14) (2003) 144112 .

[13] J. Soler, E. Artacho, J. Gale, A. García, J. Junquera, P. Ordejón, D. Sánchez-Portal, Journal of Physics: Condensed Matter 14 (11) (2002) 2745

[14] J. Perdew, K. Burke, M. Ernzerhof, Physical review letters 77 (18) (1996) 3865.

[15] G. Vérité, F. Willaime, C. Fu, in: Solid State Phenomena, Vol. 129, Trans Tech Publ, 2007, pp. 75-81.

[16] A. Lloyd, D. Cornil, A. van Duin, D. van Duin, R. Smith, S. Kenny, J. Cornil, D. Beljonne, Surface Science 645 (2016) 67-73.

[17] Y. Kim, B. Lee, M. Baskes, Physical Review B 74 (1) (2006) 014101.

[18] W. Joost, S. Ankem, M. Kuklja, Modelling and Simulation in Materials Science and Engineering 23 (1) (2014) 015006

[19] V. Trinite, Ph.D. thesis, Ecole Polytechnique X (2006).

[20] J. Murray, Phase diagrams of binary titanium alloys, ASM International, 1987, (1987) 354.

[21] L. Vitos, A. Ruban, H. Skriver, J. Kollar, The surface energy of metals, Surface Science 411 (1) (1998) 186-202. 